\title{
Enhanced Spin Coherence while Displacing Electron in a Two-Dimensional Array of Quantum Dots
}

\author{
Pierre-André Mortemousque $\odot,{ }^{1,2,},{ }^{*}$ Baptiste Jadot $\odot,{ }^{1,2}$ Emmanuel Chanrion $\odot,{ }^{1}$ Vivien Thiney $\odot,{ }^{1}$ \\ Christopher Bäuerle $\odot,{ }^{1}$ Arne Ludwig, ${ }^{3}$ Andreas D. Wieck $\odot,{ }^{3}$ Matias Urdampilleta, ${ }^{1}$ and \\ Tristan Meunier ${ }^{1}$ \\ ${ }^{1}$ Université Grenoble Alpes, CNRS, Grenoble INP, Institut Néel, Grenoble 38000, France \\ ${ }^{2}$ Université Grenoble Alpes, CEA, Leti, Grenoble F-38000, France \\ ${ }^{3}$ Lehrstuhl für Angewandte Festkörperphysik, Ruhr-Universität Bochum, Universitätsstraße 150, Bochum \\ D-44780, Germany
}

(Received 5 February 2021; accepted 8 July 2021; published 20 August 2021)

\begin{abstract}
The ability to shuttle coherently individual electron spins in arrays of quantum dots is a key procedure for the development of scalable quantum information platforms. It allows the use of sparsely populated electron spin arrays, envisioned to efficiently tackle the one- and two-qubit gate challenges. When the electrons are displaced in an array, they are exposed to site-dependent environment interactions such as hyperfine coupling with substrate nuclear spins. Here, we demonstrate that the electron multidirectional displacement in a $3 \times 3$ array of tunnel-coupled quantum dots enhances the spin-coherence time via the motional narrowing phenomenon. More specifically, up to ten charge configurations are explored by the electrons to study the impact of the displacement on spin dynamics. An increase of the coherence time by a factor up to 10 is observed in the case of fast and repetitive displacement. A simple model quantitatively captures the physical mechanism underlying this enhancement of the spin-coherence time induced by displacement. The implications on spin-coherence properties during the electron displacement are discussed in the context of large-scale quantum circuits.
\end{abstract}

DOI: 10.1103/PRXQuantum.2.030331

\section{INTRODUCTION}

Control over the flow of electrons in semiconductor circuits is core to the success of micro- and nanoelectronics. In their quantum counterparts, similar processes are investigated at the single-particle level, to preserve the fragile quantum information stored in individual electron spins [1]. Indeed, displacing these electron spins coherently opens up possibilities to convey on-chip quantum information and increase the connectivity of spin-based semiconductor quantum circuits [2-7]. Several strategies have recently been demonstrated to preserve spin coherence while shuttling charged particles. A first example consists of the confinement of electrons in moving quantum dots $[8,9]$. Another protocol is based on repetitive coherent spin tunneling between adjacent dots. Such a strategy is particularly appealing for architecture based on

\footnotetext{
*pierre-andre.mortemousque@cea.Fr
}

Published by the American Physical Society under the terms of the Creative Commons Attribution 4.0 International license. Further distribution of this work must maintain attribution to the author(s) and the published article's title, journal citation, and DOI. large two-dimensional (2D) arrays of quantum dots [10,11] and has been demonstrated for linear [12-14] and circular [15] arrays. Therefore, understanding the mechanisms at play affecting the spin-coherence preservation during electron displacements in 2D arrays is an important task to optimize this quantum information conveyer procedure and increase the distance over which the spin transfer is coherent as much as possible.

For an electron spin in a quantum dot, the loss of coherence is driven by spin dephasing mechanisms that arise from intrinsic properties of the semiconducting nanostructures, such as $1 / f$ noise coupled to spin-orbit interaction [16] or hyperfine interaction [17]. In both cases, the electron spins are experiencing slow effective magnetic field fluctuations, inducing uncertainty in the Larmor precession of individual electron spins. Therefore, refocusing techniques are extremely efficient on spin systems [18-21]. With this property in mind, displacing electron spins on fast time scales is expected to enhance the spin-coherence times by virtue of the averaging of the magnetic field fluctuations, as demonstrated in NMR via a phenomenon called the motional narrowing effect $[22,23]$. The unprecedented level of control recently demonstrated in a 2D array of quantum dots [24] permits us to displace the electrons 


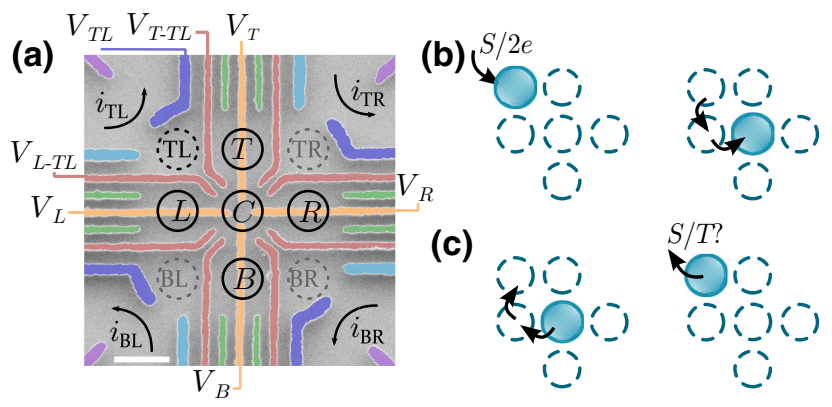

FIG. 1. The sample and spin loading and readout sequence. (a) An electron micrograph of a sample similar to the one used in this work. The nine circles indicate the $3 \times 3$ array of quantum dots (QDs) arising from the gate-induced potential landscape (see text). The five dots $L, B, R, T$, and $C$ in a crossbar configuration (solid circles) are used for the coherent electron spin shuttling in two dimensions. The square lattice of QDs is electrostatically defined due to the red gate voltages and its outer edges by the green, light blue, and blue gate voltages. The couplings of the array to electron reservoirs are achieved through the corner QDs TL, BL, BR, and TR and by controlling the light blue and blue gates. The purple gates are used to define four quantum point contacts operated as local electrometers, the conductances of which set the measured currents $i_{\mathrm{TL}}, \mathrm{BL}, \mathrm{BR}, \mathrm{TR}$. The scale bar (white) is $200 \mathrm{~nm}$. (b),(c) Schematics of the sequence used to load a singlet spin in $C$ (b) and to read out the final spin state after manipulation (c). through an important set of possible dot configurations on a time scale faster than the coherence time. This array turns out to be a relevant platform to investigate and reveal the consequences of electron displacement on spin-coherence properties.

Here, we study the coherence of individual electron spins when they are displaced via tunneling through a $2 \mathrm{D}$ array of five quantum dots defined in an $(\mathrm{Al}, \mathrm{Ga})$ As heterostructure. In this displacement regime, with an electron speed below $100 \mathrm{~ms}^{-1}$, the hyperfine interaction with the nuclei of the semiconductor nanostructure is responsible for the loss of coherence [22,25]. This hyperfine interaction results in an effective fluctuating magnetic field, called the Overhauser field, with a distribution imposed by the number of nuclei with which the electron spins are interacting. The larger the number of nuclei, the longer is the coherence time, with a typical square-root dependence [26]. During the electron displacement, the system visits a number of dot configurations imposed by the gatevoltage sequence. We relate an effective local magnetic field to each dot configuration. Two principal parameters are influencing the coherence time of the displaced electron spins: (i) the number of effective local magnetic field configurations explored, which can be varied by changing the number of accessible quantum dots in the array; and (ii) the typical interaction time fixed by the time spent in (a)

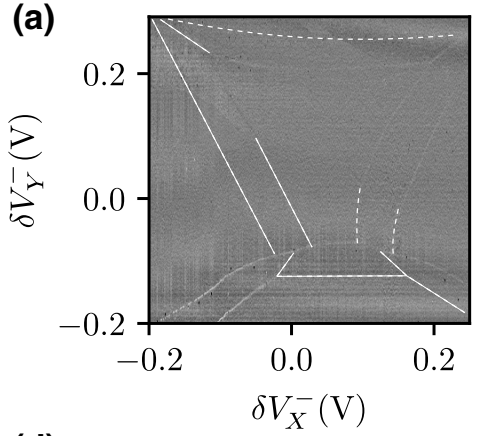

(d)

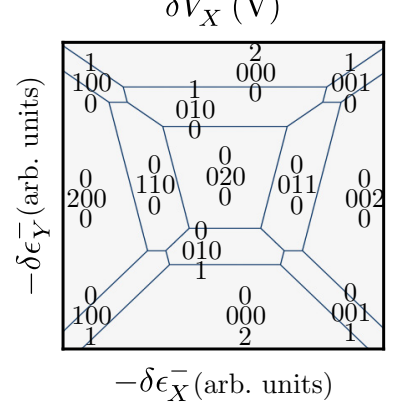

(b)

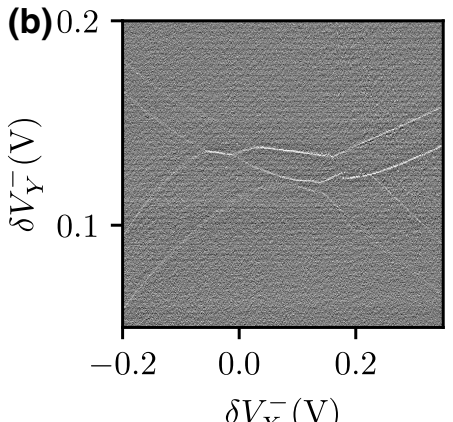

(e)

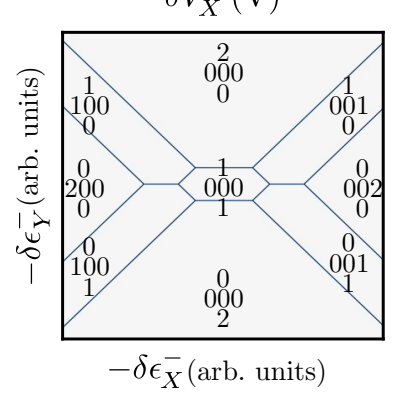

(c)

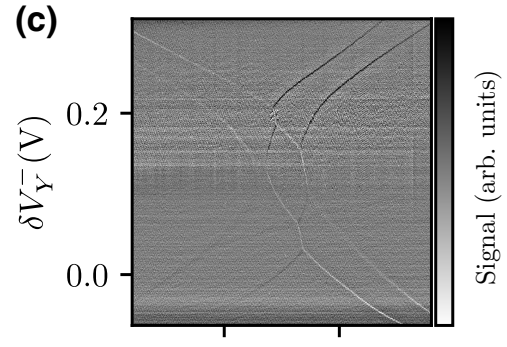

$0.1 \quad 0.2$

(f)

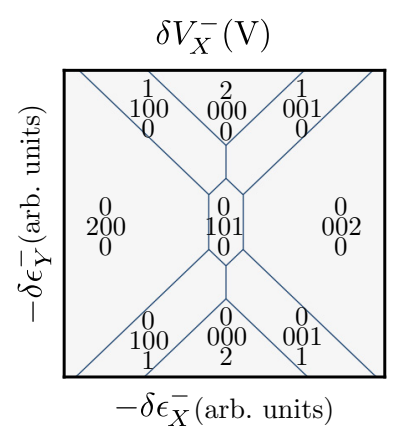

FIG. 2. Two-electron stability diagrams. (a)-(c) Charge-stability diagrams of two electrons in the five dots $L, B, R, T$, and $C$ (a) or in the four dots $L, B, R$, and $T$ (b),(c). The charge state of the array of dots is controlled by sweeping $\delta V_{X, Y}^{-}$. The signal is recorded as linear combinations of the quantum point contact current derivatives: (a),(c) $\partial_{V_{Y}^{-}} i_{\mathrm{TL}}+i_{\mathrm{BL}}$ and (b) $\partial_{V_{Y}} i_{\mathrm{TL}}+i_{\mathrm{BL}}-i_{\mathrm{BR}}$. The dashed white lines in (a) are a guide for the eye for weak-intensity degeneracy lines. The stability diagrams are obtained for different gate-voltage tunings. (d)-(f) Simulations of the stability diagrams. The labels indicate the position of the isolated electron in the QD array. 

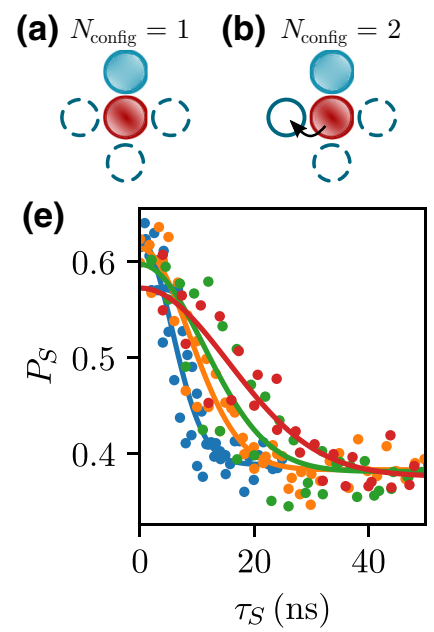
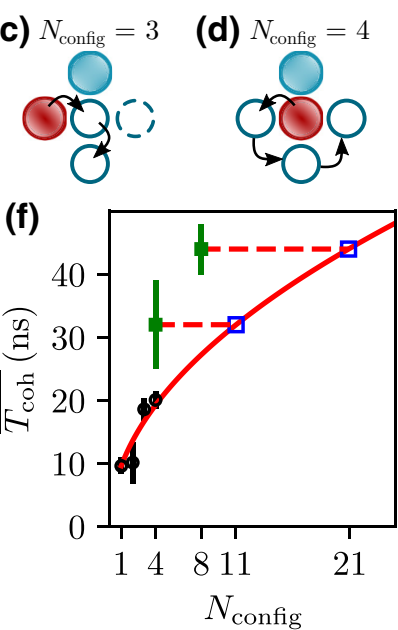

FIG. 3. Quasistatic electron spin coherence. (a)-(d) Schematics of the different electron spin displacement sequences inside the 2D array of five QDs employed to probe the electron spin coherence shown in (e). (e) The singlet probability plotted as a function of the total electron-separation time $\tau_{S}$ spent in one [T-C, blue, (a)], two [T-C and $T-L$, orange, (b)], three $[T-L, T-$ $C$, and $T-B$, green, (c)], and four [ $T-C, T-L, T-B$, and $T-R$, red, (d)] different charge configurations. The two electrons are initialized in the singlet state in the $C$ dot. Then, one electron is first transferred to $T$ and the second electron visits each other dot only once over an equal time period of $\tau_{R}$. As a consequence, for the different sequences, the second electron spends a total separation time $\tau_{S}=1 \times \tau_{R}$ in $C, \tau_{S}=2 \times \tau_{R}$ in $C$ and $L, \tau_{S}=3 \times \tau_{R}$ in $L, C$, and $B$, or $\tau_{S}=4 \times \tau_{R}$ in $C, L, B$, and $R$, where $\tau_{R}$ is in integer value of the arbitrary waveform generator (AWG) clock period. The data are fitted with Gaussian decays (solid lines) with characteristic times equal to $7.9 \pm 0.6$ (blue), $12.3 \pm 0.7$ (orange), $15.8 \pm 1.5$ (green), and $21.9 \pm 1.6 \mathrm{~ns}$ (red). (f) The coherence times averaged over different possible charge configurations $\overline{T_{\text {coh }}}$ are plotted as functions of $N_{\text {config }}$ (black circles). The data are fitted with a square-root function (red solid line) $\overline{T_{2}^{\star}} \sqrt{N_{\text {config }}}$, with $\overline{T_{2}^{\star}}=9.6 \pm 0.7 \mathrm{~ns}$. The experimental data of Figs. 5(c) and 5(d) are plotted as green squares ( $T_{\text {coh }}$ of $32 \pm 7$ and $44 \pm 4 \mathrm{~ns}$, respectively), and their projection on the squareroot curve as open blue squares (equivalent $N_{\text {config }}$ values of 11 and 21 , respectively).

each charge configuration. The demonstrated control of the dot system enables us to vary both parameters precisely and to study their impacts on the coherent spin transfer.

\section{ELECTRON SHUTTLING}

The sample [24], shown in Fig. 1(a), is an array of $3 \times 3$ laterally defined quantum dots. The device is fabricated using a Si-doped ( $\mathrm{Al}, \mathrm{Ga}) \mathrm{As} / \mathrm{GaAs}$ heterostructure grown by molecular-beam epitaxy, with a $2 \mathrm{D}$ electron gas (2DEG) $100 \mathrm{~nm}$ below the crystal surface, which has a carrier mobility of $10^{6} \mathrm{~cm}^{2} \mathrm{~V}^{-1} \mathrm{~s}^{-1}$ and an electron density of $2.7 \times 10^{11} \mathrm{~cm}^{2}$. The quantum dots are defined using electrostatic confinement generated using Ti/Au Schottky

gates. It is anchored to the cold finger, which is in turn mechanically attached to the mixing chamber of a homemade dilution refrigerator with a base temperature of 60 $\mathrm{mK}$. Quantum dots are defined and controlled by applying negative voltages on $\mathrm{Ti} / \mathrm{Au}$ Schottky gates deposited on the surface of the crystal. The dots are labeled according to their position in the array (e.g., "TL" denotes the top-left dot). The charge configurations can be read out by four quantum point contacts, tuned to be sensitive local electrometers and independently biased with $300 \mu \mathrm{V}$. The resulting currents $i_{\mathrm{TL}}, i_{\mathrm{BL}}, i_{\mathrm{BR}}$, and $i_{\mathrm{TR}}$ are measured using current-to-voltage converters with a typical bandwidth of $10 \mathrm{kHz}$. The single-shot repetition rate is about $20 \mathrm{~Hz}$.

The system is operated in a mode where the three corner dots, TR, BR, and BL, are intentionally inaccessible to the electrons (tuned at a higher potential than the crossbar dots $L, B, R, T$, and $C$ ). In this configuration, it is possible to load and isolate two electrons from the reservoir in the TL dot and to transfer them into the $\operatorname{dot} C$ by increasing the $V_{L, B, R, T}$ voltages before increasing the potential of the TL dot [Fig. 1(b)]. First, we prove that all the 15 expected charge states for two electrons in five dots $\left(\left(\begin{array}{l}5 \\ 1\end{array}\right)=5\right.$ where both electrons are in the same dot and $\left(\begin{array}{l}5 \\ 2\end{array}\right)=10$ where electrons are separated in different dots) are accessible by tuning the four linear combinations $\delta V_{X, Y}^{ \pm}$of the $V_{L, B, R, T}$ voltages (Appendix A). Figures 2(a)-2(c) show chargestability diagrams of two electrons recorded as functions of $\delta V_{X, Y}$, which act like in-plane electric dipole gates, for different values of $\delta V_{X, Y}^{+}$. By simultaneously adjusting $\delta V_{X, Y}^{+}$, it is possible to change the relative potential of the dot $C$ so that the central region of the diagrams either contains two electrons [Fig. 2(a)] or no electron, revealing the regions where the electrons can be separated in $(T, B)$ [Fig. 2(b)] and $(L, R)$ [Fig. 2(c)]. Figures 2(d)-2(f) show simulations [24] of the stability diagrams shown in Figs. 2(a)-2(c), respectively, in which the identification of the charge distributions is confirmed. In our earlier report on the coherent control of electron spin in this structure [24], we have shown that all charge states exhibited in the stability diagrams of Fig. 2 can be accessed while setting sufficiently high tunnel couplings for the coherent spin transfers between dots. Up to ten charge configurations where the electrons are separated in different dots are explored and multidirectional and complex one- and two-electron displacements are performed.

\section{COHERENT ELECTRON SPIN SHUTTLING IN A 2D ARRAY}

We investigate the two-electron spin coherence while the electrons are displaced within the $(L, B, R, T, C)$ subset of quantum dots. Before all coherent spin shuttling, two electrons in a singlet state are loaded in TL [24], before being transferred to $L$ and then $C$ [Fig. 1(b)]. Next, 


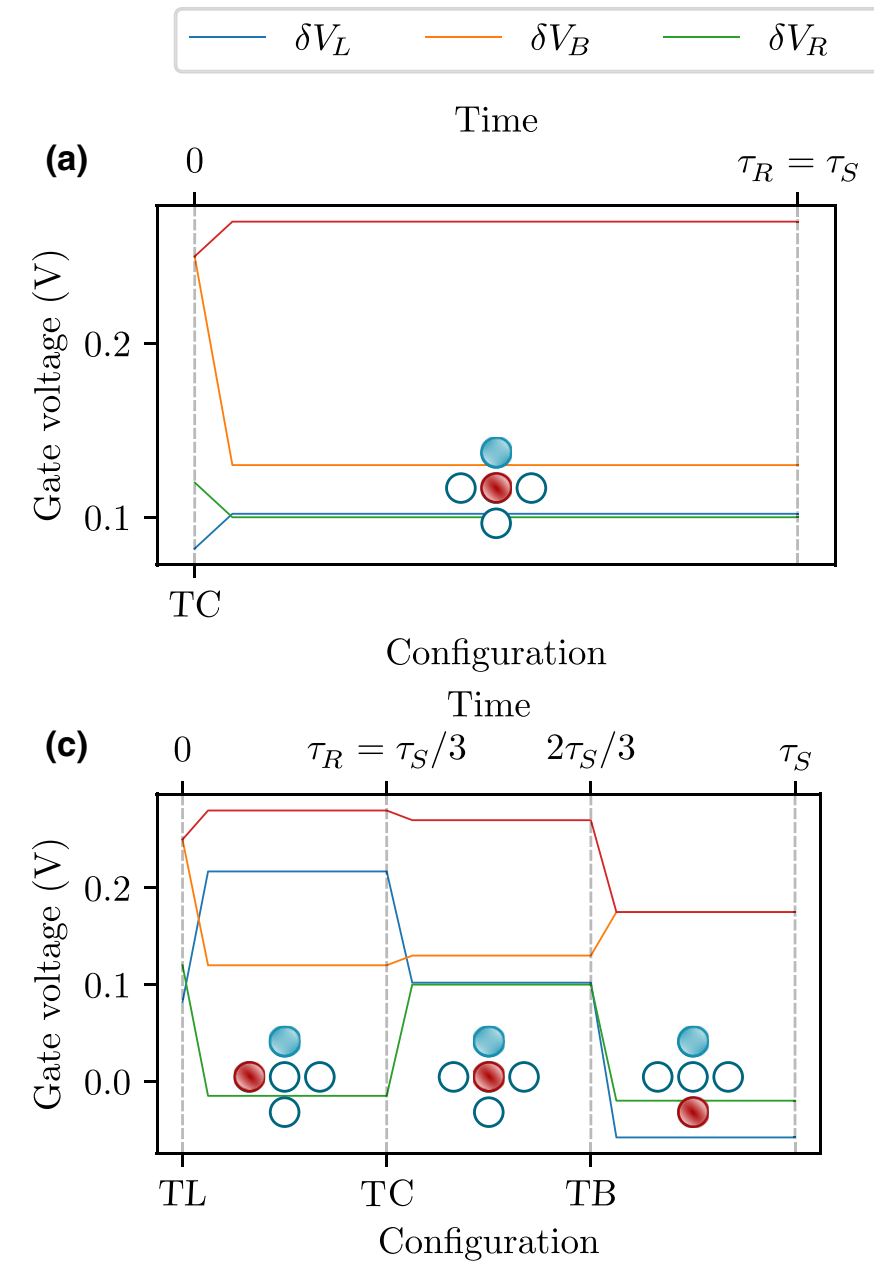

$-\delta V_{T}$
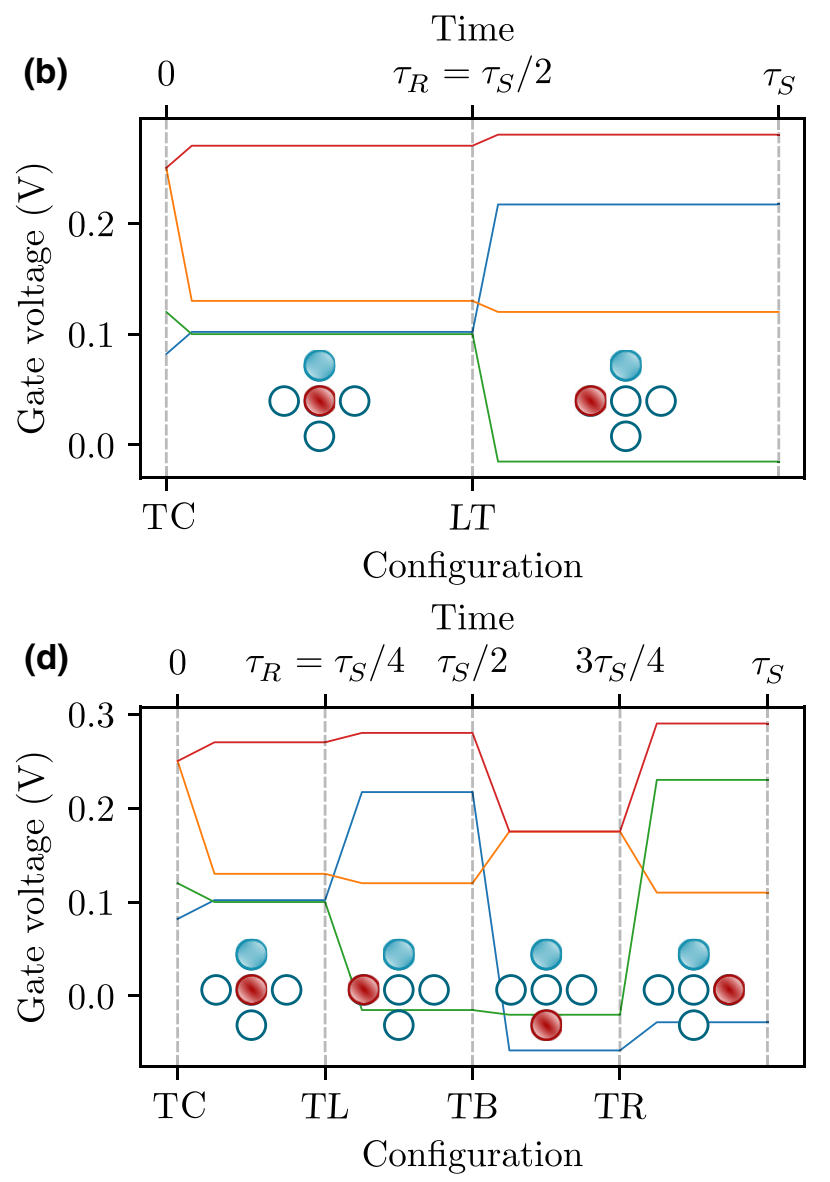

FIG. 4. Single-period electron-displacement sequences. (a)-(d) Sequences of nanosecond voltage pulses used to perform the singleperiod electron displacement of Fig. 3. (a) The pulses are applied during time $\tau_{S}$ to displace the electrons in the TC configuration. (b) The pulses are applied during time $\tau_{S} / 2$ to displace the electrons in the TC and LT configurations. (c) The pulses are applied during time $\tau_{S} / 3$ to displace the electrons in the LT, TC, and BT configurations. (d) The pulses are applied during time $\tau_{S} / 4$ to displace the electrons in the TC, LT, BT, and RC configurations. The $x$-axis labels denote the location of the dot occupied by the electron. For example, "TC" denotes one electron in $\operatorname{dot} T$ and one in $\operatorname{dot} C$. The initial and final configurations correspond to the two electrons in the $C$ dot. The schematic representations of the charge configurations are superimposed. Only one period of displacement is shown for each sequence. The voltage rise times are given as illustrations.

a sequence of voltage pulses $\delta V_{L, B, R, T}$ is used to separate and displace the electrons coherently in the array. By shuttling one or two electrons within the dot array, the separated electrons will experience decoherence, resulting in spin mixing with the triplet states. Finally, the two electrons are recombined in $C$ and transferred to $L$ and TL for spin readout [Fig. 1(c)]. To consider the three vectorial components of the hyperfine field equally, the experiments are performed without an externally applied magnetic field $[15,26]$.

We first investigate the quasistatic case, where the electrons are separated in different dots, one electron remaining in one dot while the other visits, for a single time, an increasing number of dots. For a first electron fixed in $\operatorname{dot} T$, this results in $N_{\text {config }}=1, \ldots, 4$ distinct possible configurations [Figs. 3(a)-3(d), respectively]. The corresponding voltage-pulse sequences are depicted in Fig. 4. For each experiment, the time spent in each configuration, referred to as the resting time $\tau_{R}$, is varied. The resulting singlet probabilities are plotted (filled circles) in Fig. 3(e) as a function of the total separation time $\tau_{S}=N_{\text {config }} \times \tau_{R}$. The coherence time $T_{\text {coh }}$ is directly extracted by fitting a Gaussian decay $e^{-\left(\tau_{S} / T_{\mathrm{coh}}\right)^{2}}$ (solid lines) and can be compared to the spin-coherence time $T_{2}^{\star}$ when the two electrons are separated in a double dot. We observe an increase in

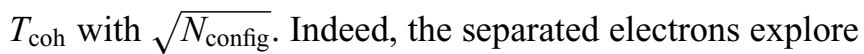
a large ensemble of nuclear spins during the displacement, averaging the hyperfine interaction [26-28] (Appendix B). We repeat this set of experiments for different permutations 
of QDs to extract a statistical dependence of $T_{\text {coh }}$ on $N_{\text {config }}$ and average out the influence of the nonregular coupling between the different dots [24]. For each size of visited subsets, the averaged $T_{\text {coh }}$, computed for three $\left(N_{\text {config }}=\right.$ $1)$, nine $\left(N_{\text {config }}=2\right)$, four $\left(N_{\text {config }}=3\right)$, and four $\left(N_{\text {config }}=\right.$ 4) trajectories, is plotted in Fig. 3(f) as a function of $N_{\text {config }}$ (the error bars correspond to the standard deviations). These values fit quantitatively with the expected squareroot law (the solid line). This confirms the importance of the hyperfine interaction and the number of nuclei with which the electrons interact. It also demonstrates the successful coherent displacement within the 2D array of dots.

\section{ENHANCEMENT OF THE SPIN-COHERENCE TIME VIA ELECTRON SHUTTLING}

We now analyze the impact of electron tunneling on their spin coherence. In comparison with the previous case where the electrons explore a single time distinct charge configurations, we study the situation where the charge configurations are periodically explored. For each experiment, the resting time spent in each charge configuration $\tau_{R}$ is set to a constant value. We use two periodic pulse sequences corresponding to two electrondisplacement patterns: (i) single- and (ii) two-electron periodic displacement. In the single-electron periodic displacement case, the first electron is maintained in $L$ and the second is displaced among $B, C, T$, and $R$, so that four isochronous dot configurations are periodically visited [Figs. 5(a) and 6(a)]. In the two-electron periodic displacement case, an electron is first moved in $B$ and the second in $R$. From this point, each electron is sequentially displaced along a two-step trajectory, either vertically or horizontally. Eight isochronous QD configurations are periodically visited, with $C$ being the only dot visited by both electrons [Figs. 5(b) and 6(b)]. The experimental singlet probabilities (solid black points) recorded for the single-electron $\left(\tau_{R}=1.7 \mathrm{~ns}\right)$ and for the two-electron $\left(\tau_{R}=2.1 \mathrm{~ns}\right)$ periodic displacement cases, are plotted in Figs. 5(c) and 5(d), respectively. The curves are fitted by exponential decays [Fig. 7(a) and 7(b)], with characteristic times of $32 \pm 7$ and $44 \pm 4 \mathrm{~ns}$, corresponding to coherence lengths of $1.9 \pm 0.4$ and $2.2 \pm 0.2 \mu \mathrm{m}$ (assuming a conservative interdot distance of $100 \mathrm{~nm}$ ), respectively. These decay times are plotted as green squares in Fig. 3(f), as is their projection on the square-root curve as open blue squares $\left(N_{\text {config }}\right.$ values equivalent to 11 and 21, respectively). A significant improvement is observed compared to the quasistatic case: these values are 1.6 times higher than the expected coherence times for four- and eight-static-dot configurations, respectively. Moreover, the decoherence law changes from Gaussian-like [Fig. 3(e)] to exponential [Figs. 5(c) and 5(d)] decay when visiting the charge configuration multiple times, which confirms the significant impact of the electron dynamics on their spin coherence.
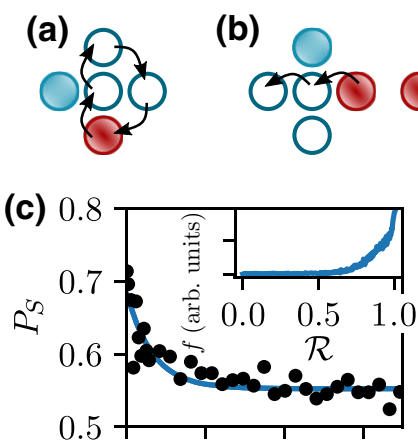

(d)
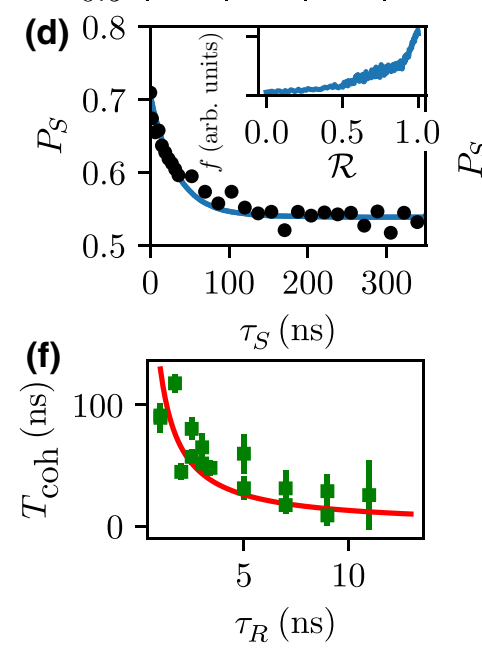

(b)

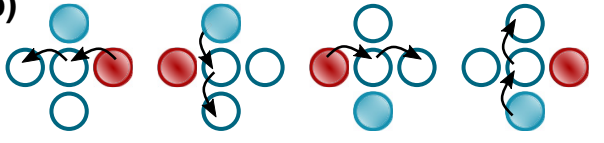

(e)

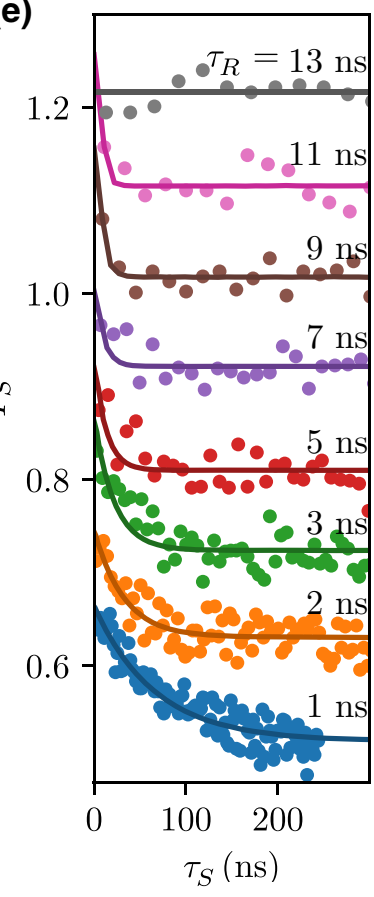

FIG. 5. The enhanced spin-coherence time for periodic electron displacements. (a)-(b) Schematics of the different electron spin periodic displacement sequences inside the $2 \mathrm{D}$ array of five QDs employed to probe the electron spin coherence shown in (c)-(f). (c),(d) Singlet probabilities plotted (black dots) as a function of the total electron-separation time $\tau_{S}$ (c) for the single-electron periodic displacement pattern (a), and (d) for the two-electron periodic displacement pattern (b). The data are acquired for configuration durations $\tau_{R}$ of $1.7 \mathrm{~ns}$ (c) and $2.1 \mathrm{~ns}$ (d). The experimental data are fitted using simulations of the spin dynamics (see text) as solid blue lines. The fit error functions (see text) are shown in insets. (e) The singlet probability plotted (dots) as a function of the total electron-separation time $\tau_{S}$, for increasing (bottom to top) values of $\tau_{R}=1,2,3,5,7,9$, 11 , and $13 \mathrm{~ns}$, for the two-electron periodic displacement case (b). The solid lines are the simulated singlet probabilities (see text) computed for the corresponding $\tau_{R}$ times, for a common remanence value $\mathcal{R}=0$. (f) The characteristic coherence times $T_{\text {coh }}$ plotted as functions of $\tau_{R}$ and fitted with an inverse function $\left(T_{2}^{\star}\right)^{2} / \tau_{R}+T_{2}^{\star}$, with $T_{2}^{\star}=11.4 \pm 4.8 \mathrm{~ns}$.

By varying the resting time $\tau_{R}$, we can explore different shuttling regimes for the two-electron periodic displacement case. The resulting singlet probability is plotted in Fig. $5(\mathrm{e})$ as a function of $\tau_{S}=\left(8 N_{\text {cycle }}+1\right) \tau_{R}$ for different values of $\tau_{R}$ going from $1 \mathrm{~ns}$ to $13 \mathrm{~ns}$ (bottom to top). No singlet probability decay is observed for $\tau_{R}$ longer than the static spin-coherence time due to complete mixing between the singlet and triplet states. By progressively reducing $\tau_{R}$, 


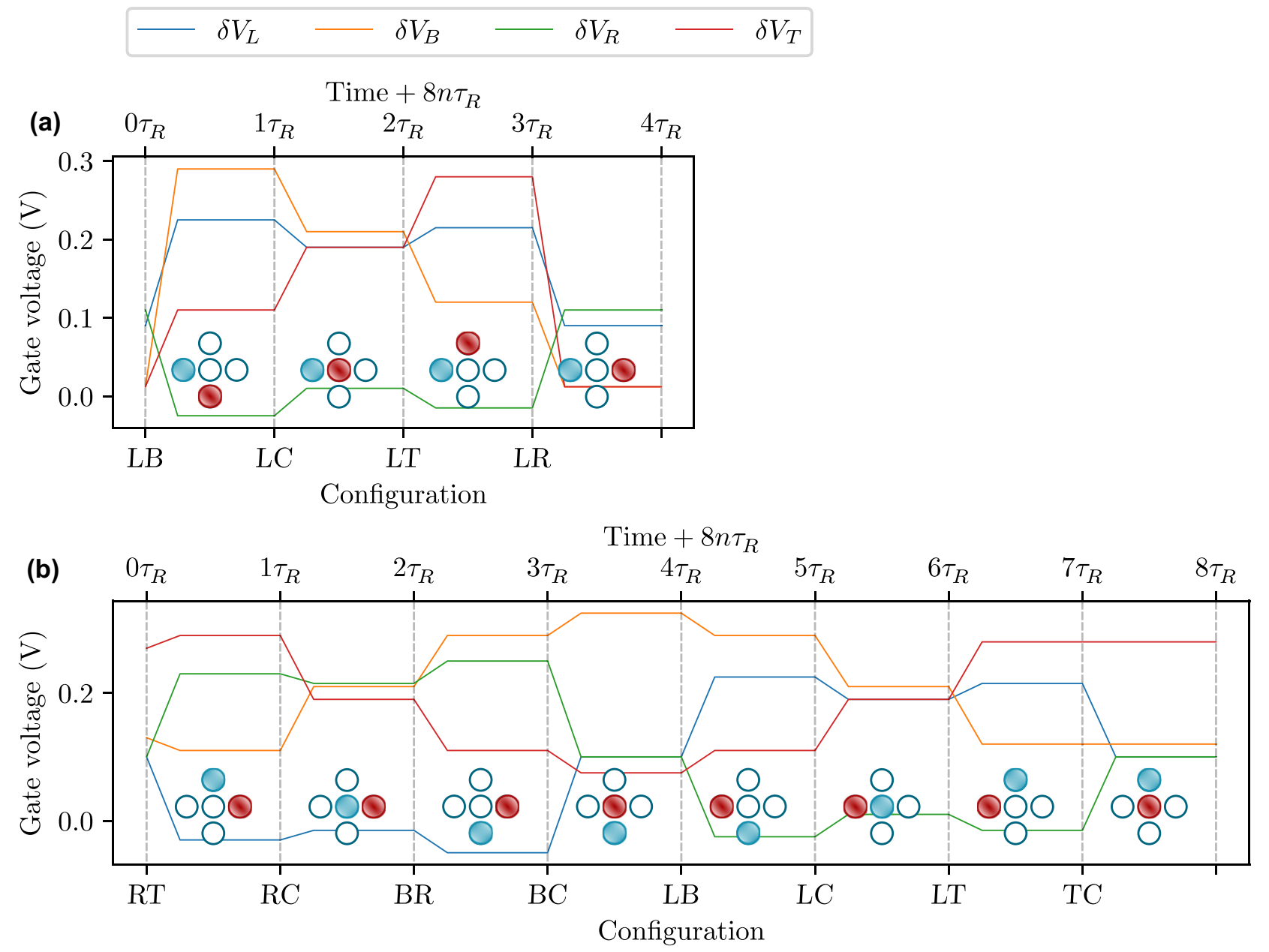

FIG. 6. Periodic electron-displacement sequences. (a),(b) Sequences of nanosecond voltage pulses used to perform the periodic electron displacements. (a) One electron remains in the left quantum dot while the second electron is displaced in the $B, C, T$, and $R$ dots. (b) One electron periodically visits $L, C$, and $R$ and the second $T, C$, and $B$. The $x$-axis labels denote the location of the dot occupied by the electron. For example, "LB" denotes one electron in dot $L$ and one in dot $B$. The schematic representations of the charge configurations are superimposed. Only one period of displacement is shown for each sequence. The voltage rise times are given as illustrations.

we observe the emergence of a single exponential decay of the singlet probability, demonstrating the preservation of spin coherence during displacement. We extract the characteristic decay time $T_{\text {coh }}$ by fitting with exponential decay [Fig. 7(c)], which we plot against $\tau_{R}$ [Fig. 5(f)]. The points are fitted with an inverse function $\left(T_{2}^{\star}\right)^{2} / \tau_{R}+T_{2}^{\star}$, typical of a motional narrowing phenomenon [23], with a dephasing time $T_{2}^{\star}=11.4 \pm 4.8 \mathrm{~ns}$. This value is in agreement with the dephasing time observed in our experiment in Fig. 3(f) of $\overline{T_{2}^{\star}}=9.6 \pm 0.7 \mathrm{~ns}$.

\section{PATH RANDOMIZATION}

If only a definite set of dot configurations is cyclically explored, no difference in the spin dynamics should be observed between the quasistatic and the periodic displacement protocols. Then, the only relevant parameter is the time spent in each configuration and a Gaussian decay of the singlet probabilities would be expected. However, the difference observed in the experiment forces us to reconsider the initial assumptions made about the electron displacement: the electrons are not periodically exploring the same set of random magnetic field configurations. To better understand the impact of electron shuttling on the spin-coherence time, we model the system and study the spin dynamics (Fig. 8 and Appendix C). We introduce the notion of randomization of the effective hyperfine field during the coherent electron displacements. It takes the form of a remanent parameter $0 \leq \mathcal{R} \leq 1$ and it describes the change in effective hyperfine field, between two consecutive displacement periods $i$ and $i+1$, at equivalent dot $j$. It is expressed as $\vec{B}_{i+1}^{j}=\mathcal{R} \vec{B}_{i}^{j}+(1-\mathcal{R}) \overrightarrow{\delta B}_{i+1}^{j}$, where $\overrightarrow{\delta B}_{i+1}^{j}$ is a random occurrence of the magnetic field 

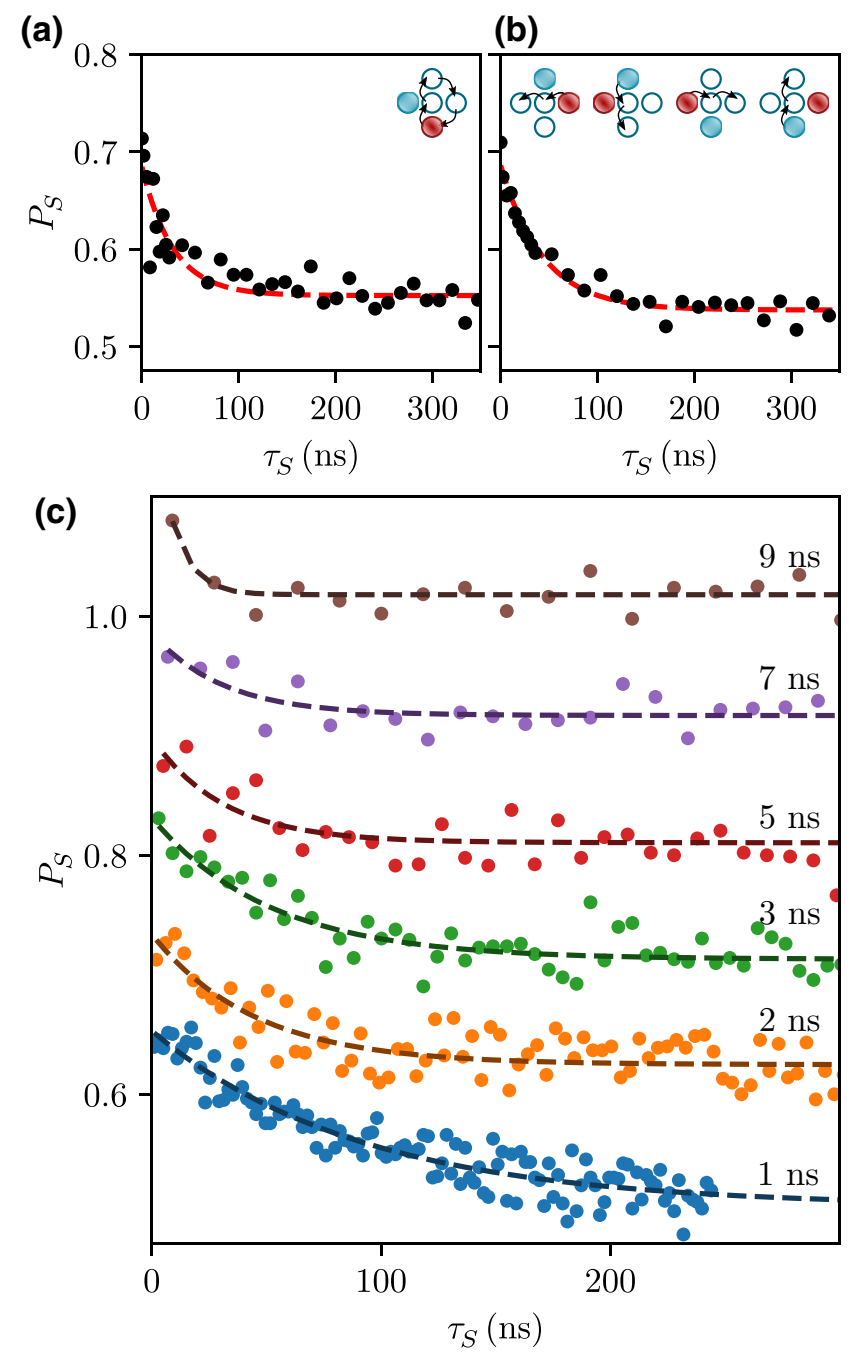

FIG. 7. Exponential spin-coherence fits of the periodically displaced electron. In order to extract the coherence times $T_{\text {coh }}$ that are discussed in the text, we fit the experimental data using a single exponential decay law. (a),(b) The same data set as in Figs. 5(c) and 5(d), respectively. The data are fitted with an exponential decay (dashed lines) with characteristic times of (a) $32 \pm 7$ and (b) $44 \pm 4$ ns (red). (c) The same data set as in Fig. 5(e). The data are fitted with an exponential decay (dashed lines) with the following characteristic times: $91.3 \pm 10$ (blue), $44.8 \pm 7$ (orange), $51.7 \pm 8$ (green), $31.3 \pm 9$ (red), $31.2 \pm 15$ (purple), and $8.9 \pm 9$ (brown).

distribution. For example, a full remanence $\mathcal{R}=1$ means that the effective hyperfine interaction for an electron visiting the same dot multiple times is constant over the entire displacement sequence of a single shot. Assuming that (i) the evolution of the effective hyperfine field is much slower than the coherent spin displacement [30,31], (ii) the nuclear spin is immune to the electrostatic manipulation, and (iii) it is not affected by the electron motion, the remanence value is then interpreted as a small electrostatic potential reorganization, leading to fluctuations

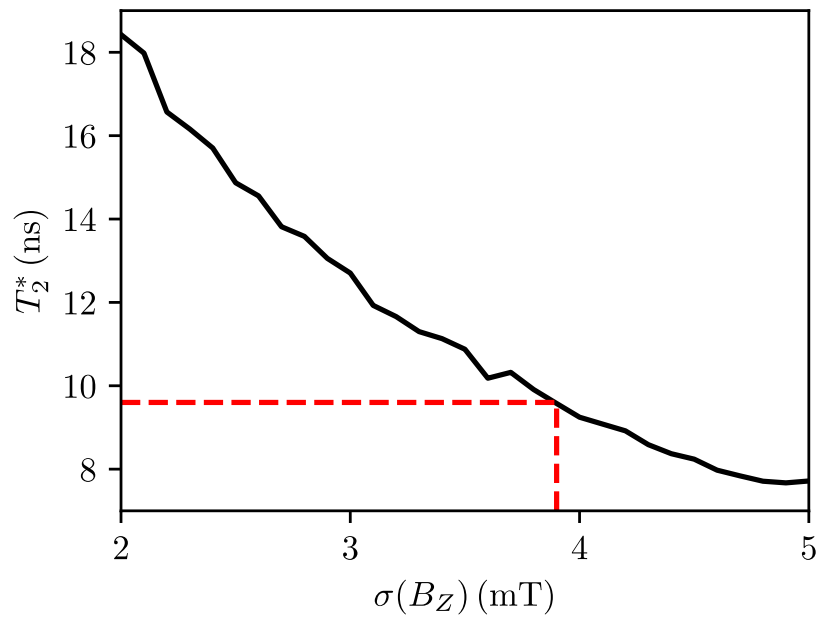

FIG. 8. The simulated spin-coherence time in a static double quantum dot. The electron spin decoherence curves can be simulated for different standard deviation $\sigma\left(B_{Z}\right)$ values of a Gaussian distribution in hyperfine fields. The coherence times extracted from Gaussian fits are plotted as functions of $\sigma\left(B_{Z}\right)$. The field corresponding to the spin-coherence time $T_{2}^{\star}=9.6 \mathrm{~ns}$ [extracted from the $T_{2}^{\star}\left(\sqrt{N_{\text {config }}}\right)$ fit of Fig. 3(f)] is pinpointed with a dashed red line $\left(B_{Z}=3.9 \mathrm{mT}\right)$.
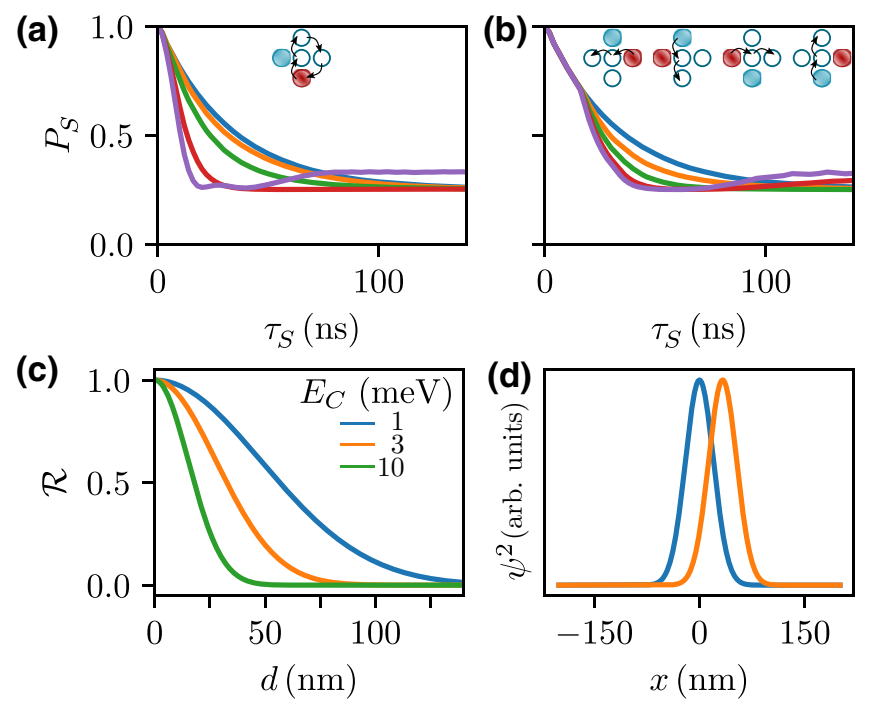

FIG. 9. The consequences of electron-path randomization. (a),(b) Simulated decoherence decay curves for (a) one and (b) two displaced electrons for different values of the remanence parameter $\mathcal{R}$ : 0 (blue), 0.5 (orange), 0.7 (green), 0.9 (red), and 1 (purple). (c) The estimated remanence $\mathcal{R}$ plotted as a function of the distance between the dot locations of two consecutive periods, for a charging energy of 1 (blue), 3 (orange), and $10 \mathrm{meV}$ (green). (d) A cross-section view of the two-electron densities computed using the fundamental Fock-Darwin state [29] and separated by $33 \mathrm{~nm}(\mathcal{R}=0.5)$, assuming a charging energy of $3 \mathrm{meV}$. 


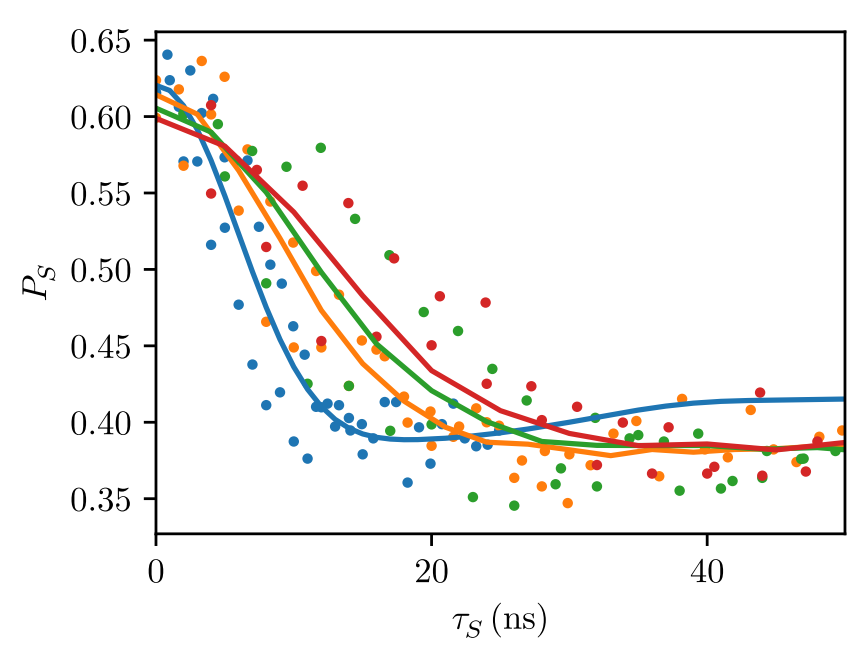

FIG. 10. The simulated decays of the quasistatic electron spin coherence, using the same data set as in Fig. 3(e). The experimental data (dots) are compared with the simulations (solid lines) of the coherence spin in the quasistatic case (the remanence parameter is irrelevant here, as each configuration is explored only a single time). The overshoot in the singlet probability described in Ref. [26] is observed in the simulations only for the double quantum dot case (blue) and is not observed in the experimental data. For the two electrons in three (orange), four (green), or five (red) dots, the simulated decays are Gaussian-like.

in the dot positions. For $\mathcal{R}<0.5$, the decoherence decay curves are exponential-like [see Figs. 9(a) and 9(b)] and only weakly dependent on $\mathcal{R}$, which is the fingerprint of a continuous motional narrowing effect. On the other hand, for $\mathcal{R}>0.5$, the motional-narrowing process is limited by the number of visited sites, which leads to Gaussian or Gaussian-like [26] coherence decays. Such a transition from Gaussian to exponential dynamics is a clear manifestation of the motional-narrowing effect that has been observed in liquid NMR.

The simulation reproduces well the experimental data in the single- and two-electron displacement with a remanence parameter $\mathcal{R} \approx 0$ [solid blue curves in Figs. 5(c) and 5(d)]. The evaluation of the best-fitting simulated coherence decay curves (calculated to minimize the sumof-squares difference with the experimental data; see the insets) show that the data from the two experiments can be reproduced with a remanence value of $0 \lesssim \mathcal{R}<0.5$. One can note that the difference in decay times between the single- and the two-electron-displacement cases is not significant with regard to the fit uncertainties [Figs. 7(a) and 7(b)]. Moreover, this parameter also provides simulated coherence decays that are very similar to the experimental data of Fig. 5(e), for the various values $\tau_{R}$ of the displacement dynamics (solid lines), as well as for the data obtained in the quasistatic experiments (Fig. 10).

Therefore, the cyclic nature of the evolution has to be randomized along the path of the electrons to reproduce the experimental data. Since the hyperfine interaction is a contact interaction, a displacement of the size of the dot will be sufficient to change the effective magnetic field completely. The relationship between $\mathcal{R}$ and the electron center-of-mass separation between two consecutive periods $d$ is given for various charging energies in Fig. 9(c). It is calculated as the overlap between the electron density at two consecutive periods $\mathcal{R}=\int \psi_{i}^{2} \psi_{i+1}^{2} d r$. Therefore, between two consecutive displacement periods, the electron center of mass is expected to be distant by about $d \approx 33 \mathrm{~nm}$ for a charging energy of $3 \mathrm{meV}$ [Figs. 9(d) and 11]. The schematic electron center-of-mass trajectories in the single- and the two-electron-displacement experiments are superimposed on the gate pattern and the simulated electrostatic potential of Figs. 11(a) and 11(b), respectively. Moreover, the limited number of displacement cycles for which the electron spins are in phase prevents us from observing any hyperfine field correlation when the electrons revisit the same quantum dots.

Here, we speculate that these fluctuations in dot positions may occur because of the large voltage ranges employed to displace the electron through the quantum dot array (as large as a few hundreds of millivolts; see Fig. 6). Such energy values are expected to induce perturbations of the semiconductor nanostructure, resulting in a change in the disorder potential. Additionally, the electrostatic potential simulations of Figs. 11(a) and 11(b) confirm that the confinement potentials remain shallow during the electron shuttling, making the dot positions very sensitive to fluctuations of charges in the substrate (e.g., donors in the doping layer of the heterostructure; see Fig. 12). Moreover, the typical time scale of this alteration is comparable to that of the excitation potential and therefore can be as fast as $1 \mathrm{~ns}$. It implies that the electrons are displaced along a path that is fluctuating on a time scale comparable to the time needed to explore a few dot configurations. As a consequence, the effective magnetic field configuration is no longer precisely cyclic and becomes randomized.

A similar assumption with regard to the electron path has been reported recently by our group in a different displacement regime [9]. The electrons were then displaced in moving quantum dots at a speed of $3000 \mathrm{~m} / \mathrm{s}$. Indeed, at this speed, the motional-narrowing process is extremely efficient for hyperfine interaction and the main mechanism for decoherence is mediated by spin-orbit interaction. Evidence of change in the disorder potential is imprinted in the coherence of the transported electrons, which are initially prepared in a superposition of antiparallel spin states.

\section{CONCLUSIONS}

We perform one- and two-electron coherent shuttling in a 2D array and explore various displacement trajectories through different sets of quantum dots. We report 

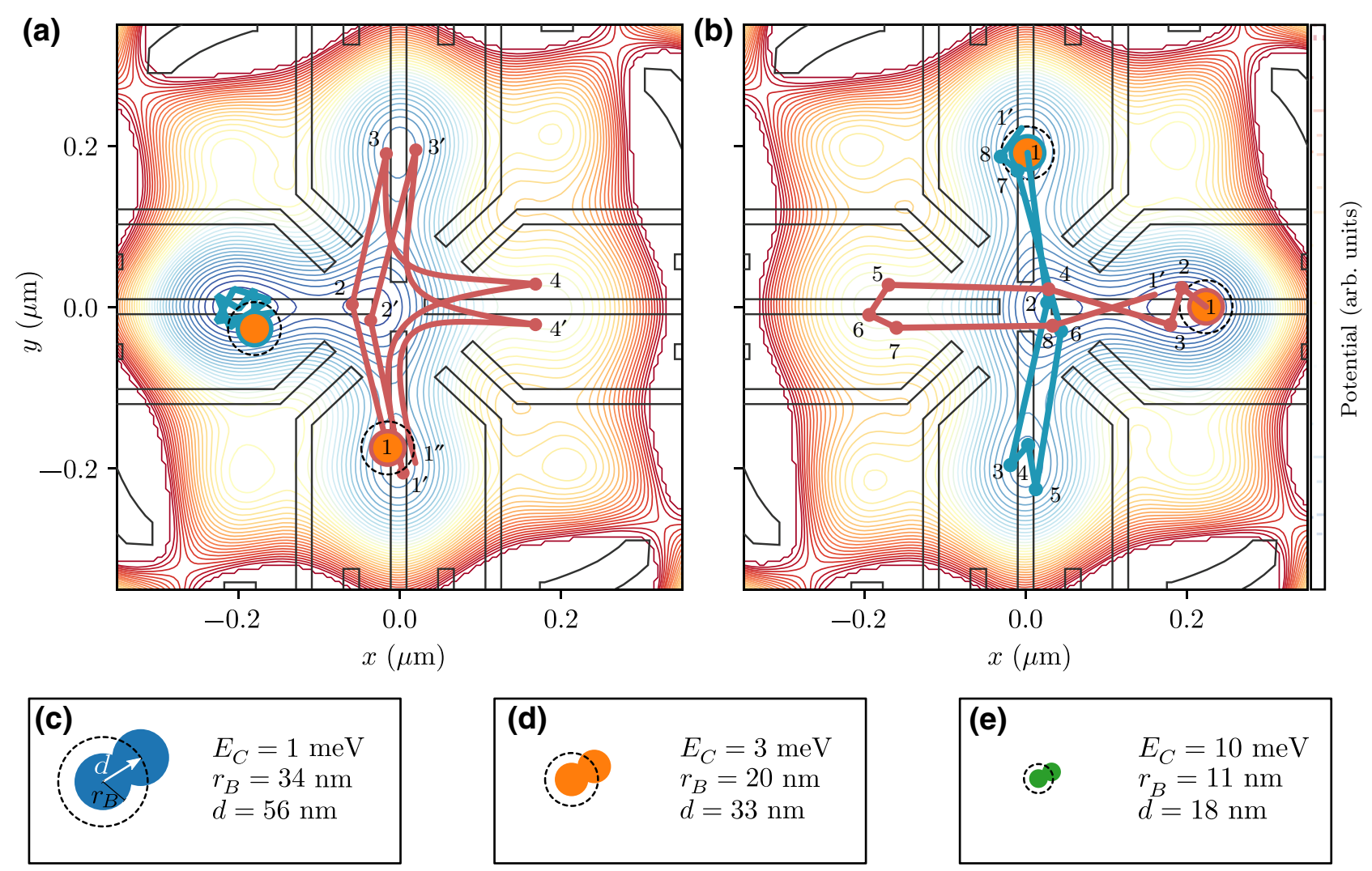

FIG. 11. Electrostatic potential simulations. (a),(b) Electrostatic potential simulations performed with gate-voltage configurations corresponding to the confinement of two electrons (a) in $L-B$ and (b) in $T-R$. Hypothetical electron trajectories (dots) are overlapped for the two displacement patterns considered. The solid lines are guides for the eye. (a) A single electron is periodically displaced between dots $(B, C, T$, and $R$ ), while the other electron remains in the $L$ dot. Four dot configurations are explored, but the dot positions evolve at each period (two periods are drawn, e.g., $\left.1 \rightarrow 1^{\prime} \rightarrow 1^{\prime \prime}\right)$. (b) Two electrons are displaced, one between $(L, C, R)$ and the other between $(T, C, B)$. Eight dot configurations are explored and, here again, the dot positions evolve at each displacement period (a single period is drawn, e.g., $1 \rightarrow 1^{\prime}$ ). The electrostatic potential simulations are performed by solving the Laplace equation [35]. The orange circles have a radius of $20 \mathrm{~nm}$, corresponding to the Bohr radius of an electron with a charging energy $E_{C}$ of $3 \mathrm{meV}$. The dashed circles have a radius of $33 \mathrm{~nm}$, a value computed to reach a remanence $\mathcal{R}=0.5$. (c)-(e) Schematic representations of the Bohr radii and corresponding dot displacements to reach a remanence $\mathcal{R}=0.5$ for different electron charging energies.

an increase of the coherence time for the two-electron displacements by a factor of 13 , which corresponds to an increase of the coherence length above $12 \mu \mathrm{m}$. Furthermore, we specifically study the zero-magnetic-field regime, where all the three components of the hyperfine interaction contribute equally to the decoherence of the two electron spin singlet states. This hyperfine interaction is expected to be averaged during the electron displacement. A signature of a motional-narrowing process is observed, with a decoherence rate inversely proportional to the time spent in the static phase. Indeed, reducing the time where the electrons are effectively static and averaging faster than the spin dynamics over many nuclear spin configurations increases the observed coherence time [22]. An important consequence of the observation is the signature that the path of the electron is slightly modified from one period of displacement to the next.
This results in the randomization of the effective field distribution experienced by the electrons. The experimental results are reproduced using a simple model. Therefore, only the single-electron displacement during the gate movement from one dot to another is relevant to explain the increase in the spin-coherence time. This protocol, which consists of probing the spin coherence as a function of the number of displacement steps, can in principle be applied to other sources of magnetic field inhomogeneity, such as micromagnets near quantum dots or $g$-factor perturbation in chains of quantum dots, to assess the reproducibility of the qubit manipulation and transport in arrays of dots or quantum channels. Finally, as the estimated remanent hyperfine field is low, our experiment is analogous to the displacement of an electron in a linear chain of quantum dots. The observed increase in the spin-coherence time induced by motional narrowing lets us envision the 


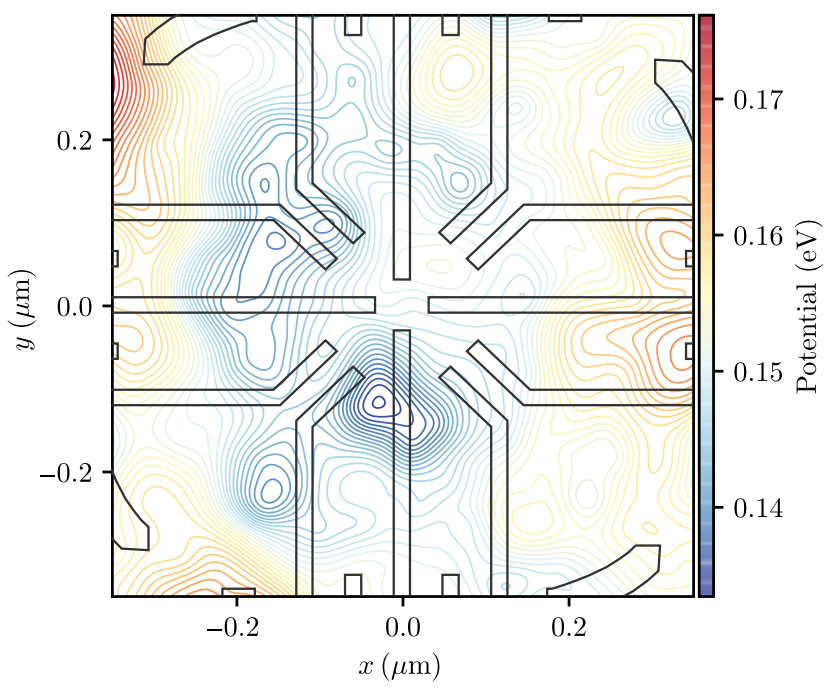

FIG. 12. Simulation of the electrostatic potential arising from a random distribution of donors. The potential is computed for a random distribution of donors in the Si-doped layer of the heterostructure that produces a $2 \mathrm{DEG}$ with an electron density of $2.7 \times 10^{11} \mathrm{~cm}^{2}$. The induced potential can localize the dots in different spatial positions than the one intended by the gate geometry.

long-range coherent displacement of spins in semiconductors (even isotopically engineered) along statistically imperfect channels or chains of dots.

\section{ACKNOWLEDGMENTS}

We would like to thank Maud Vinet, Xuedong Hu, and Lieven M. K. Vandersypen for enlightening discussions. We acknowledge technical support from the Poles of the Institut Néel, and in particular, the NANOFAB team, who helped with the sample realization, as well as E. Eyraud, T. Crozes, P. Perrier, G. Pont, H. Rodenas, D. Lepoittevin, C. Hoarau, and C. Guttin. M.U. acknowledges the support of project CODAQ (ANR-16-ACHN0029). A.L. and A.D.W. acknowledge gratefully the support of DFG-TRR160, BMBF-Q.com-H 16KIS0109, and the DFH/UFA CDFA-05-06. T.M. acknowledges financial support from ERC QSPINMOTION, ERC QUCUBE, ANR CMOSQSPIN (Grant No. ANR-17-CE24-0009), ANR SiQuBus, UGA IDEX (Grant No. ANR-15-IDEX02), and H2020 QLSI.

\section{APPENDIX A: DEFINITION OF THE VIRTUAL GATES}

The gate-voltage control of the five central dots is best understood using a set of virtual gates acting like in-plane multipoles rather than the physical gate voltages. The inplane $x$ and $y$ electric dipolelike gates are denoted $\delta V_{X, Y}^{-}$.
To complete the set of gates, we also use $\delta V_{X, Y}^{+}$:

$$
\left[\begin{array}{c}
\delta V_{X}^{+} \\
\delta V_{X}^{-} \\
\delta V_{Y}^{+} \\
\delta V_{Y}^{-}
\end{array}\right]=\left[\begin{array}{cccc}
1 & 1 & 0 & 0 \\
-1 & 1 & 0 & 0 \\
0 & 0 & 1 & 1 \\
0 & 0 & -1 & 1
\end{array}\right]\left[\begin{array}{l}
\delta V_{L} \\
\delta V_{R} \\
\delta V_{B} \\
\delta V_{T}
\end{array}\right] .
$$

Finally, the voltage applied on each gate is the elementwise sum of the $\delta V_{L, B, R, T}$ values and the offset values $V_{L, B, R, T}^{0}$. Typical voltage offsets of $\left(V_{L}^{0}, V_{B}^{0}, V_{R}^{0}, V_{T}^{0}\right)=$ $(-0.38,-0.27,-0.37,-0.29) \mathrm{V}$, as used for Fig. 2(b), are employed.

\section{APPENDIX B: NUCLEAR SPIN DYNAMICS AND EXPERIMENTAL TIME SCALES}

In this appendix, we describe the acquisition protocol employed in this work. The voltage control apparatus is programmed to execute and repeat a list of single-shot experiments (about a few thousand different sequences). Homemade electronics ensure fast changes of both chemical potentials and tunnel couplings with voltage-pulse rise times approaching $100 \mathrm{~ns}$ and refreshed every $16 \mu \mathrm{s}$. This is supplemented by a Tektronix 5014C AWG, with a typical channel-voltage rise time $(20 \%-80 \%)$ of $0.9 \mathrm{~ns}$, used to rapidly change the $V_{L}, V_{B}, V_{R}$, and $V_{T}$ gate voltages. The fast slew rate of the AWG channels ensures a reproducible electron displacement even for short resting times. Moreover, no change in the coherence time of the electron spins due to a modulation in the dot potential detunings (corresponding to a gate-voltage settling time from $80 \%$ to $100 \%$ of the target values) is observed in this experiment.

The total duration of each single shot (comprising the electron spin initialization, manipulation, readout, and the instrumental overhead) is about $50 \mathrm{~ms}$. The experimental singlet probabilities are calculated as the average spin readout results of either 150 [Fig. 5(e)] or 1000 single shots [Figs. 3(e), 5(c) and 5(d)]. Therefore, the typical time between the repetition of two equivalent sequences is about a few minutes, which is much longer than the nuclear spin relaxation time in the dipole-dipole field of other nuclear spins, which is in the range of $100 \mu \mathrm{s}$ $[26,32]$. This allows the nuclear spin distribution to be randomized between each equivalent single-shot experiment. Consequently, there is no preferential quantification axis at zero magnetic field and the spin-orbit interactions are averaged out.

\section{APPENDIX C: SIMULATION OF THE ELECTRON SPIN DYNAMICS}

The simulations are performed with an initial singlet spin state. A four-level system is considered to model the two electron spins, each exposed to a specific 
magnetic field (random hyperfine interaction with the substrate nuclear spins). The numerical time integration of the Schrödinger equation is computed for 1000 different couples of magnetic field vectors, following a centered Gaussian distribution with a standard deviation of 3.9 $\mathrm{mT}$ (see Fig. 8). Magnetic-field-remanence parameters are added to the model to consider the spatial evolution of the effective dot locations during the different displacement patterns. Finally, the average singlet probability is calculated $[33,34]$.

[1] G. Burkard and D. Loss, in Semiconductor Spintronics and Quantum Computation (Springer-Verlag, Berlin, 2002).

[2] J. M. Taylor, H.-A. Engel, W. Dur, A. Yacoby, C. M. Marcus, P. Zoller, and M. D. Lukin, Fault-tolerant architecture for quantum computation using electrically controlled semiconductor spins, Nat. Phys. 1, 177 (2005).

[3] A. D. Greentree, J. H. Cole, A. R. Hamilton, and L. C. L. Hollenberg, Coherent electronic transfer in quantum dot systems using adiabatic passage, Phys. Rev. B 70, 235317 (2004).

[4] A. J. Skinner, M. E. Davenport, and B. E. Kane, Hydrogenic Spin Quantum Computing in Silicon: A Digital Approach, Phys. Rev. Lett. 90, 087901 (2003).

[5] C. H. W. Barnes, J. M. Shilton, and A. M. Robinson, Quantum computation using electrons trapped by surface acoustic waves, Phys. Rev. B 62, 8410 (2000).

[6] L. M. K. Vandersypen, H. Bluhm, J. S. Clarke, A. S. Dzurak, J. Ishihara, A. Morello, D. J. Reilly, L. R. Schreider, and $\mathrm{M}$. Veldhorst, Interfacing spin qubits in quantum dots and donors - hot, dense, and coherent, npj Quantum Inf. 3, 34 (2017).

[7] A. R. Mills, D. M. Zajac, M. J. Gullans, F. J. Schupp, T. M. Hazard, and J. R. Petta, Shuttling a single charge across a one-dimensional array of silicon quantum dots, Nat. Commun. 10, 1063 (2019).

[8] B. Bertrand, S. Hermelin, S. Takada, M. Yamamoto, S. Tarucha, A. Ludwig, A. D. Wieck, C. Bäuerle, and T. Meunier, Fast spin information transfer between distant quantum dots using individual electrons, Nat. Nanotech. 11, 672 (2016).

[9] B. Jadot, P. A. Mortemousque, E. Chanrion, V. Thiney, A. Ludwig, A. D. Wieck, M. Urdampilleta, C. Bauerle, and T. Meunier, Distant spin entanglement via fast and coherent electron shuttling, ArXiv:2004.02727 (2020).

[10] M. Vinet, et al., in 2018 IEEE International Electron Devices Meeting (IEDM) (IEEE, San Francisco, CA, USA, 2018), p. 6.5.1.

[11] R. Li, L. Petit, D. P. Franke, J. P. Dehollain, J. Helsen, M. Steudtner, N. K. Thomas, Z. R. Yoscovits, K. J. Singh, $\mathrm{S}$. Wehner, et al., A crossbar network for silicon quantum dot qubits, Sci. Adv. 4, eaar3960 (2018).

[12] T. Fujita, T. A. Baart, C. Reichl, W. Wegscheider, and L. M. K. Vandersypen, Coherent shuttle of electron-spin states, npj Quantum Inf. 3, 22 (2017).

[13] J. Yoneda, W. Huang, M. Feng, C. H. Yang, K. W. Chan, T. Tanttu, W. Gilbert, R. C. C. Leon, F. E. Hudson, K.
M. Itoh, A. Morello, S. D. Barlett, A. Laucht, A. Saraiva, and A. S. Dzurak, Coherent spin qubit transport in silicon, ArXiv:2008.04020.

[14] F. Ginzel, A. R. Mills, J. R. Petta, and G. Burkard, Spin shuttling in a silicon double quantum dot, Phys. Rev. B 102, 195418 (2020).

[15] H. Flentje, P. A. Mortemousque, R. Thalineau, A. Ludwig, A. D. Wieck, C. Bäuerle, and T. Meunier, Coherent longdistance displacement of individual electron spins, Nat. Commun. 8, 501 (2017).

[16] J. Yoneda, K. Takeda, T. Otsuka, T. Nakajima, M. R. Delbecq, G. Allison, T. Honda, T. Kodera, S. Oda, Y. Hoshi, N. Usami, K. M. Itoh, and S. Tarucha, A quantum-dot spin qubit with coherence limited by charge noise and fidelity higher than 99.9\%, Nat. Nanotechnol. 13, 102 (2018).

[17] M. Veldhorst, J. C. C. Hwang, C. H. Yang, A. W. Leenstra, B. de Ronde, J. P. Dehollain, J. T. Muhonen, F. E. Hudson, K. M. Itoh, A. Morello, and A. S. Dzurak, An addressable quantum dot qubit with fault-tolerant control-fidelity, Nat. Nanotechnol. 9, 981 (2014).

[18] A. M. Tyryshkin, S. Tojo, J. J. L. Morton, H. Riemann, N. V. Abrosimov, P. Becker, H.-J. Pohl, T. Schenkel, M. L. W. Thewalt, K. M. Itoh, and S. A. Lyon, Electron spin coherence exceeding seconds in high-purity silicon, Nat. Mater. 11, 143 (2011).

[19] M. D. Shulman, S. P. Harvey, J. M. Nichol, S. D. Bartlett, A. C. Doherty, V. Umansky, and A. Yacoby, Suppressing qubit dephasing using real-time Hamiltonian estimation, Nat. Commun. 5, 5156 (2014).

[20] G. Wolfowicz, P.-A. Mortemousque, R. Guichard, S. Simmons, M. L. W. Thewalt, K. M. Itoh, and J. J. L. Morton, ${ }^{29} \mathrm{Si}$ nuclear spins as a resource for donor spin qubits in silicon, New J. Phys. 18, 023021 (2016).

[21] H. Bluhm, S. Foletti, I. Neder, M. Rudner, D. Mahalu, V. Umansky, and A. Yacoby, Dephasing time of GaAs electron-spin qubits coupled to a nuclear bath exceeding 200 microsecond, Nat. Phys. 7, 109 (2011).

[22] P. Huang and $X$. Hu, Spin qubit relaxation in a moving quantum dot, Phys. Rev. B 88, 075301 (2013).

[23] C. P. Slichter, Principles of Magnetic Resonance (SpringerVerlag, Berlin, 1978), 3rd ed.

[24] P.-A. Mortemousque, E. Chanrion, B. Jadot, H. Flentje, A. Ludwig, A. D. Wieck, M. Urdampilleta, C. Bäuerle, and T. Meunier, Coherent control of individual electron spins in a two-dimensional quantum dot array, Nat. Nanotechnol. 16, 296 (2020).

[25] C. Echeverria-Arrondo and E. Y. Sherman, Relaxation of flying spin qubits in quantum wires by hyperfine interaction, Phys. Rev. B 87, 081410(R) (2013).

[26] I. A. Merkulov, A. L. Efros, and M. Rosen, Electron spin relaxation by nuclei in semiconductor quantum dots, Phys. Rev. B 65, 205309 (2002).

[27] H. Flentje, B. Bertrand, P. A. Mortemousque, V. Thiney, A. Ludwig, A. D. Wieck, C. Bäuerle, and T. Meunier, A linear triple quantum dot system in isolated configuration, Appl. Phys. Lett. 110, 233101 (2017).

[28] N. Bloembergen, E. M. Purcell, and R. V. Pound, Relaxation effects in nuclear magnetic resonance absorption, Phys. Rev. 73, 679 (1948). 
[29] L. P. Kouwenhoven, D. G. Austing, and S. Tarucha, Fewelectron quantum dots, Rep. Prog. Phys. 64, 701 (2001).

[30] D. J. Reilly, J. M. Taylor, E. A. Laird, J. R. Petta, C. M. Marcus, M. P. Hanson, and A. C. Gossard, Measurement of Temporal Correlations of the Overhauser Field in a Double Quantum Dot, Phys. Rev. Lett. 101, 236803 (2008).

[31] T. Nakajima, A. Noiri, K. Kawasaki, J. Yoneda, P. Stano, S. Amaha, T. Otsuka, K. Takeda, M. R. Delbecq, G. Allison, A. Ludwig, A. D. Wieck, D. Loss, and S. Tarucha, Coherence of a Driven Electron Spin Qubit Actively Decoupled from Quasistatic Noise, Phys. Rev. X 10, 011060 (2020).

[32] F. K. Malinowski, F. Martins, L. Cywinski, M. S. Rudner, P. D. Nissen, S. Fallahi, G. C. Gardner, M. J. Manfra, C. M. Marcus, and F. Kuemmeth, Spectrum of the Nuclear
Environment for GaAs Spin Qubits, Phys. Rev. Lett. 118, 177702 (2017).

[33] J. R. Johansson, P. D. Nation, and F. Nori, QUTIP: An open-source PYTHON framework for the dynamics of open quantum systems, Comput. Phys. Commun. 183, 1760 (2012).

[34] J. R. Johansson, P. D. Nation, and F. Nori, QUTIP 2: A PYTHON framework for the dynamics of open quantum systems, Comput. Phys. Commun. 184, 1234 (2013).

[35] T. Bautze, C. Süssmeier, S. Takada, C. Groth, T. Meunier, M. Yamamoto, S. Tarucha, X. Waintal, and C. Bäuerle, Theoretical, numerical, and experimental study of a flying qubit electronic interferometer, Phys. Rev. B 89, 125432 (2014). 